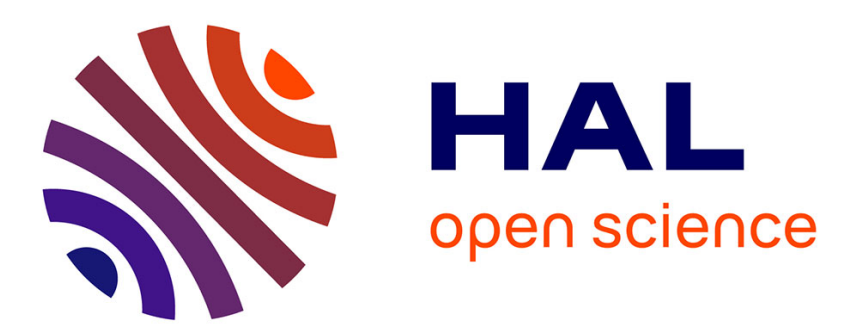

\title{
Stochastic Stability of a Class of Stochastic Bilinear Hybrid systems: Convex Analysis and Synthesis
}

Samir Aberkane, Jean-Christophe Ponsart, Dominique Sauter, Frédéric Hamelin

\section{- To cite this version:}

Samir Aberkane, Jean-Christophe Ponsart, Dominique Sauter, Frédéric Hamelin. Stochastic Stability of a Class of Stochastic Bilinear Hybrid systems: Convex Analysis and Synthesis. Aug 2006, pp.10271032. hal-00121674

\section{HAL Id: hal-00121674 \\ https://hal.science/hal-00121674}

Submitted on 21 Dec 2006

HAL is a multi-disciplinary open access archive for the deposit and dissemination of scientific research documents, whether they are published or not. The documents may come from teaching and research institutions in France or abroad, or from public or private research centers.
L'archive ouverte pluridisciplinaire HAL, est destinée au dépôt et à la diffusion de documents scientifiques de niveau recherche, publiés ou non, émanant des établissements d'enseignement et de recherche français ou étrangers, des laboratoires publics ou privés. 


\title{
STOCHASTIC STABILITY OF A CLASS OF STOCHASTIC BILINEAR HYBRID SYSTEMS: CONVEX ANALYSIS AND SYNTHESIS
}

\author{
S. Aberkane J.C. Ponsart D. Sauter F. Hamelin
}

\author{
CRAN - CNRS UMR 7039 \\ Université Henri Poincaré, Nancy 1, BP 239, \\ F-54506 Vandoeuvre-lès-Nancy Cedex \\ Tel.: +33 383684480, Fax: +33383684462 \\ e-mail: samir.aberkane@cran. uhp-nancy.fr
}

\begin{abstract}
This paper deals with the problem of local exponential stochastic stabilization of continuous time Bilinear Active Fault Tolerant Control Systems with Markovian Parameters (BAFTCSMP). The design technique is based on a differential inclusion of the bilinear term for a restricted domain of the state space. The above problematic is addressed under a convex programming approach. Indeed, conditions for local stochastic stability of the BAFTCSMP are derived and formulated through some matrix inequalities. The implicit problem being to maximize the region in which the closed-loop stability can be ensured, some convex optimization problems with LMI (linear matrix inequalities) relaxations schemes are stated. A numerical example is presented to illustrate the theoretical results.
\end{abstract}

Keywords: Active Fault Tolerant Control - Bilinear systems - Hybrid systems Markovian jumping parameters - Local stochastic stability - Positive invariance LMI.

\section{INTRODUCTION}

Active fault tolerant control systems are feedback control systems that reconfigure the control law in real time based on the response from an automatic fault detection and identification (FDI) scheme. The dynamic behaviour of active fault tolerant control systems (AFTCS) is governed by stochastic differential equations (because the failures and failure detection occur randomly) and can be viewed as a general hybrid system (Srichander and Walker 1993). A major class of hybrid systems is jump linear systems (JLS). In JLS, a single jump process is used to describe the random variations affecting the system parameters. This process is represented by a finite state Markov chain and is called the plant regime mode. The theory of stability, optimal control and $\mathcal{H}_{2} / \mathcal{H}_{\infty}$ control, as well as important applications of such systems, can be found in several papers in the current literature, for instance in (Costa et al. 1999, de Farias et al. 2000, de Souza and Fragoso 1993, Ji and Chizeck 1990, Ji and Chizeck 1992).

To deal with AFTCS, another class of hybrid systems was defined, denoted as active fault tolerant control systems with Markovian parameters (AFTCSMP). In this class of hybrid systems, two random processes are defined: the first random process represents system components failures and the second random process represents the FDI process used to reconfigure the control law. This model was proposed by Srichander and Walker (Srichander and Walker 1993). Necessary and sufficient conditions for stochastic stability of AFTCSMP were developed for a single component failure (actuator failures). Such a formulation offers a convenient framework in the consideration of delays and errors 
in detecting and isolating the failures.

On the other hand, bilinear systems (BLS) have been of great interest for the three last decades, since many real-world systems can be adequately approximated by a bilinear model. In this class of systems, some control signal $u(t)$ exerts its effect purely multiplicatively, but for a fixed $u(t)$, it exhibits a linear comportment. BLS may be intentionally designed to obtain better system response than is possible with a linear design, or they may be appropriate models for systems to be controlled (Elliott 1999). However, a literature search reveals that the issue of stabilization of continuous time Bilinear Active Fault Tolerant Control Systems with Markovian Parameters (BAFTCSMP) has not been fully investigated and remains important and challenging. This situation motivates the present study on the stochastic stabilization of BAFTCSMP.

In this paper, we are concerned with the problem of local exponential stochastic stabilization of continuous time BAFTCSMP subject to multiple failure processes: one for plant components and the other for actuators. The main reason for using two independent failure processes is that it allows the modelling of faults at different locations with independent failure characteristics (Mahmoud et al. 2003). The design technique is based on a differential inclusion of the bilinear term for a restricted domain of the state space. The above problematic is addressed under a convex programming approach. Indeed, conditions for local stochastic stability of the BAFTCSMP are derived and formulated through some matrix inequalities. The implicit problem being to maximize the region in which the closed-loop stability can be ensured, some convex optimization problems with LMI (linear matrix inequalities) relaxations schemes are stated.

This paper is organized as follows: section 2 describes the dynamical model of the system with appropriately defined random processes. A brief summary of basic stochastic terms, results and definitions are given in section 3. Section 4 derives the sufficient conditions for the local stochastic exponential stability in the mean square sense of the BAFTCSMP. A numerical example is presented in section 5 to illustrate the theoretical results. Finally, a conclusion is given in section 6 .

\section{DYNAMICAL MODEL}

The system under normal operation $(\varphi)$ can be described by:

$$
\varphi:\left\{\begin{array}{l}
\dot{x}(t)=A x(t)+B u(t)+\sum_{i=1}^{m} N_{i} x(t) u_{i}(t) \\
y(t)=C_{2} x(t)
\end{array}\right.
$$

where $A \in \mathbb{R}^{n \times n}, B \in \mathbb{R}^{n \times r}, C_{2} \in \mathbb{R}^{q \times n}, x(t) \in \mathbb{R}^{n}$ is the system state, $u(t) \in \mathbb{R}^{r}$ is the system input and $y(t) \in \mathbb{R}^{q}$ is the system measured output.
It is important to note that a basic point to determine the appropriate dynamical model which describes the faulty system is the location of a fault and the nature of the faulty components. In this paper, we will consider that the system is subject to both plant components and actuator failures. The random changes affecting plant components are represented by a homogeneous Markov process $\xi(t)$ with the finite state space $Z=\{1,2, \ldots, z\}$, and the random changes that occur in actuators are represented by another homogeneous Markov process $\eta(t)$ with the finite state space $S=\{1,2, \ldots, s\}$. In practice, these random variations are not directly measurable but rather can only be monitored by an FDI scheme. Let $\psi(t)$ denote the state of the FDI process which monitors the states $\xi(t)$ and $\eta(t)$ of the random processes describing the failures. The process $\psi(t)$ is a finite state stochastic process whose random behaviour is conditioned on the failures processes states $\eta(t)$ and $\xi(t)$, therefore, the state space of the FDI process $\psi(t)$ contains the state spaces of the two failure processes. This state space is also finite and is denoted by $R=\{1,2, \ldots, r\}$.

In AFTCS, we consider that the control law is only a function of the mesurable FDI process $\psi(t)$. Therefore, the BAFTCSMP can be described as:

$$
\varphi:\left\{\begin{array}{l}
\dot{x}_{t}=A\left(\xi_{t}\right) x_{t}+B\left(\eta_{t}\right) u\left(x_{t}, \psi_{t}, t\right)+\sum_{i=1}^{m} N_{i}\left(\xi_{t}, \eta_{t}\right) x_{t} u_{i}\left(x_{t}, \psi_{t}, t\right) \\
y_{t}=C_{2} x_{t} \\
u\left(x_{t}, \psi_{t}, t\right)=\mathcal{K}\left(\psi_{t}\right) x_{t}
\end{array}\right.
$$

For notational simplicity, we will denote $A\left(\xi_{t}\right)=A_{i}$, when $\xi_{t}=i \in Z, B\left(\eta_{t}\right)=B_{j}$ when $\eta_{t}=j \in S$, and $\mathcal{K}\left(\psi_{t}\right)=\mathcal{K}_{k}$ when $\psi_{t}=k \in R$. We also denote $\bullet(t)=\bullet_{t}$ and the initial conditions $\bullet\left(t_{0}\right)=\bullet_{0}$.

The FDI and the Failure Processes: $\xi_{t}, \eta_{t}$ and $\psi_{t}$ being homogeneous Markov processes with finite state spaces, we can define the transition probability of the plant components failure process as:

$$
\begin{cases}p_{i j}(\Delta t)=\pi_{i j} \Delta t+o(\Delta t) & (i \neq j) \\ p_{i i}(\Delta t)=1-\sum_{i \neq j} \pi_{i j} \Delta t+o(\Delta t) & (i=j)\end{cases}
$$

The transition probability of the actuator failure process is given by:

$$
\begin{cases}p_{k l}(\Delta t)=\nu_{k l} \Delta t+o(\Delta t) & (k \neq l) \\ p_{k k}(\Delta t)=1-\sum_{k \neq l} \nu_{k l} \Delta t+o(\Delta t) & (k=l)\end{cases}
$$

where $\pi_{i j}$ is the plant components failure rate, and $\nu_{k l}$ is the actuator failure rate. Given that $\xi_{t}=k$ and $\eta_{t}=l$, the conditional transition probability of the FDI process $\psi_{t}$ is:

$$
\begin{cases}p_{i v}^{k l}(\Delta t)=\lambda_{i v}^{k l} \Delta t+o(\Delta t) & (i \neq v) \\ p_{i i}^{k l}(\Delta t)=1-\sum_{i \neq v} \lambda_{i v}^{k l} \Delta t+o(\Delta t) & (i=v)\end{cases}
$$

Here, $\lambda_{i v}^{k l}$ represents the transition rate from $i$ to $v$ for the Markov process $\psi_{t}$ conditioned on $\xi_{t}=k \in Z$ and $\eta_{t}=l \in S$. 


\section{DEFINITIONS}

Under the assumption that the system $(\varphi)$ satisfies the global Lipschitz condition, the solution $x_{t}$ determines a family of unique continuous stochastic processes, one for each choice of the random variable $x_{0}$. The joint process $\left\{x_{t}, \xi_{t}, \eta_{t}, \psi_{t}\right\}$ is a Markov process.

For system (2), we have the following definitions:

Definition 1 (Local stochastic Stability): System (2) is said to be locally exponentially stable in the mean square sense if for any initial conditions $\left(x_{0}, \xi_{0}, \eta_{0}, \psi_{0}\right)$ there exists two numbers $a>0$ and $b>0$ and a subset $\mathcal{D}_{0}$ in $\mathbb{R}^{n}$ such that when $x_{0} \in \mathcal{D}_{0}$, the following inequality holds $\forall t \geq t_{0}$ for all solution of (2) with initial condition $x_{0}$ :

$$
\mathcal{E}\left\{\left\|x_{t}\right\|^{2}\right\} \leq b\left\|x_{0}\right\|^{2} \exp \left[-a\left(t-t_{0}\right)\right]
$$

Definition 2 (Positive Invariance): A subset $\mathcal{D}_{0}$ in $\mathbb{R}^{n}$ is said to be positive invariant with respect to (w.r.t) the system (2), if, for any initial condition $x_{0} \in \mathcal{D}_{0}$, the trajectory $x\left(x_{0}, \xi_{t}, \eta_{t}, \psi_{t}, t\right) \in \mathcal{D}_{0}$, $\forall t \geq 0$, and $\forall \xi_{t} \in Z, \eta_{t} \in S$ and $\psi_{t} \in R$.

We conclude this section by recalling the following lemma which will be useful for the proof of our main results in the next sections.

Lemma 1 (Reciprocal Projection Lemma) (Apkarian et al. 2001): Let $P$ be any given positive-definite matrix. The following statements are equivalent:

1) $\Psi+S+S^{T}<0$

2) the LMI problem

$$
\left[\begin{array}{cc}
\Psi+P-\left(W+W^{T}\right) & S^{T}+W^{T} \\
S+W & -P
\end{array}\right]<0
$$

is feasible with respect to $W$.

\section{MAIN RESULTS}

In this section, we will derive sufficient conditions for local exponential stability in the mean square sense of the system (2). The design technique is based on a differential inclusion of the bilinear term for a restricted domain of the state space.

\subsection{Local Stochastic Stability}

The problematic addressed in this section can be formulated as follows:

Problem 1: Find feedback gains $\mathcal{K}\left(\psi_{t}\right) \in \mathbb{R}^{m \times n}$ and a region of stability (a set of initial conditions) $\mathcal{D}_{0}$ such that the system (2) is locally exponentially stabilized in the mean square sense by $u_{t}=\mathcal{K}\left(\psi_{t}\right) x_{t}$ for any initial condition $x_{0}$ belonging to $\mathcal{D}_{0}$. $\diamond$ To solve problem 1 , we have to determine a domain $\mathcal{D}_{0}$ such that the closed loop system:

$$
\varphi_{c l}:\left\{\begin{array}{l}
\dot{x}_{t}=A\left(\xi_{t}\right) x_{t}+N\left(x_{t}, \xi_{t}, \eta_{t}\right) \mathcal{K}\left(\psi_{t}\right) x_{t} \\
y_{t}=C_{2} x_{t} \\
N\left(x_{t}, \xi_{t}, \eta_{t}\right)=B\left(\eta_{t}\right)+\left[N_{1}\left(\xi_{t}, \eta_{t}\right) x_{t} \ldots N_{m}\left(\xi_{t}, \eta_{t}\right) x_{t}\right]
\end{array}\right.
$$

is locally exponentially stable in the mean square sense, $\forall x_{0} \in \mathcal{D}_{0}$. In order to determine both suitable stabilizing gains $\mathcal{K}\left(\psi_{t}\right)$ and a domain of stability $\mathcal{D}_{0}$, we can consider that the state is restricted to belong to a certain compact domain in $\mathbb{R}^{n}, \forall \xi_{t} \in Z, \eta_{t} \in S$ and $\psi_{t} \in R$, described by:

$$
\Psi(x)=\left\{x \in \mathbb{R}^{n} ; \mathcal{Q} x \leq \kappa\right\}
$$

where $\mathcal{Q} \in \mathbb{R}^{g \times n}, n \leq g, \operatorname{rank} \mathcal{Q}=n$, and $\kappa_{i}>0$, $\forall i=1, \ldots, g$. The positivity of vector $\kappa$ means that the origin belongs to the interior of $\Psi(x)$. Furthermore, we suppose that $\Psi(x)$ is a compact set and therefore it satisfies:

$$
\Psi(x) \subseteq\left\{x \in \mathbb{R}^{n}, x=\sum_{j=1}^{q} \mu_{j} X_{j}, \sum_{j=1}^{q} \mu_{j}=1, \mu_{j} \geq 0\right\}
$$

$\forall \xi_{t} \in Z, \eta_{t} \in S$ and $\psi_{t} \in R$.

or equivalently:

$$
\Psi(x) \subseteq \operatorname{Co}\left\{X_{j}, j=1, \ldots, q\right\}, \quad \forall \xi_{t} \in Z, \eta_{t} \in S, \psi_{t} \in R
$$

Remark 1: In (7), the vectors $X_{j} \in \mathbb{R}^{n}$ are the vertices allowing to describe the convex hull containing the points of $\Psi(x)$.

Hence a way to exhibit solutions to problem 1 is to search both $\mathcal{K}\left(\psi_{t}\right)$ and $\mathcal{D}_{0} \subseteq \Psi(x)$. An implicit problem is then to be able to maximize the size of the domain of stability $\mathcal{D}_{0}$.

\subsection{Differential Inclusion}

The main idea to solve problem 1 is to express a local equivalent representation of the bilinear term of system (2) for $x_{t} \in \Psi(x)$. Indeed, from differential inclusion results (Molchanov and Pyatniskii 1989), providing that $x_{t} \in \Psi(x)$, we get:

$\left[A\left(\xi_{t}\right)+N\left(x_{t}, \xi_{t}, \eta_{t}\right) \mathcal{K}\left(\psi_{t}\right)\right] \in \operatorname{Co}\left\{\mathcal{A}_{1}\left(\xi_{t}, \eta_{t}, \psi_{t}\right), \ldots, \mathcal{A}_{q}\left(\xi_{t}, \eta_{t}, \psi_{t}\right)\right\}$

$$
\forall \xi_{t} \in Z, \eta_{t} \in S, \psi_{t} \in R
$$

where

$$
\begin{aligned}
\mathcal{A}_{j}\left(\xi_{t}, \eta_{t}, \psi_{t}\right)=A\left(\xi_{t}\right)+ & \left(B\left(\eta_{t}\right)\right. \\
+ & {\left.\left[N_{1}\left(\xi_{t}, \eta_{t}\right) X_{j}, \ldots, N_{m}\left(\xi_{t}, \eta_{t}\right) X_{j}\right]\right) \mathcal{K}\left(\psi_{t}\right) } \\
& \forall j=1, \ldots, q
\end{aligned}
$$

Thus, if $x_{t} \in \Psi(x)$, then $\dot{x}_{t}$ can be determined from the following polytopic model:

$$
\begin{gathered}
\dot{x}_{t}=\sum_{j=1}^{q} \lambda_{j}(t) \mathcal{A}_{j}\left(\xi_{t}, \eta_{t}, \psi_{t}\right) x_{t} \\
\forall \xi_{t} \in Z, \eta_{t} \in S, \psi_{t} \in R
\end{gathered}
$$

with $\sum_{j=1}^{q} \lambda_{j}(t)=1, \lambda_{j}(t) \geq 0$.

The solution of problem 1 consists in determining positive definite functions $\mathcal{V}_{1}\left(x_{t}\right), \mathcal{V}_{2}\left(x_{t}, \xi_{t}, \eta_{t}, \psi_{t}, t\right)$, gain matrices $\mathcal{K}\left(\psi_{t}\right)$ and a set $\mathcal{D}_{0}$ such that:

1) $\mathcal{V}_{1}\left(x_{t}\right)$ and $\mathcal{V}_{2}\left(x_{t}, \xi_{t}, \eta_{t}, \psi_{t}, t\right)$ satisfie the conditions to be a Lyapunov, and a stochastic Lyapunov functions (see (Srichander and Walker 1993) for the definition of a stochastic Lyapunov function), respectively;

2) $\dot{\mathcal{V}}_{1}\left(x_{t}\right)=\frac{\partial \mathcal{V}_{1}}{\partial x} \dot{x}_{t} \leq 0, \forall \xi_{t} \in Z, \eta_{t} \in S, \psi_{t} \in R$ and $\forall x_{0} \in \mathcal{D}_{0}$

3) $\beta_{1}\left\|x_{t}\right\|^{2} \leq \mathcal{V}_{2}\left(x_{t}, \xi, \eta, \psi, t\right) \leq \beta_{2}\left\|x_{t}\right\|^{2}$;

4) $\mathcal{L} \mathcal{V}_{2}\left(x_{t}, \xi_{t}, \eta_{t}, \psi_{t}, t\right) \leq-\beta_{3}\left\|x_{t}\right\|^{2}, \forall \xi_{t} \in Z, \eta_{t} \in S, \psi_{t} \in R$, and $\forall x_{0} \in \mathcal{D}_{0}$, where $\beta_{1}, \beta_{2}, \beta_{3}$ are real positive constants; 
where $\mathcal{L}$ is the weak infinitesimal operator of the joint Markov process $\left\{x_{t}, \xi_{t}, \eta_{t}, \psi_{t}\right\}$.

In order to simplify conditions (2), we want to use the description of the term $N\left(x_{t}, \xi_{t}, \eta_{t}\right)$ described in section (4.1). These can be stated by the following theorem.

Theorem 1: If there exist a Lyapunov function $\mathcal{V}_{1}\left(x_{t}\right)$, a stochastic Lyapunov function $\mathcal{V}_{2}\left(x_{t}, \xi_{t}, \eta_{t}, \psi_{t}, t\right)$, matrices $\mathcal{K}\left(\psi_{t}\right)$ and $\mathcal{Q}$ of appropriate dimensions, vectors $X_{j}, j=1, \ldots, q$, a positive vector $\kappa$ and a positive scalar $\gamma$ satisfying

$$
\begin{aligned}
& \frac{\partial \mathcal{V}_{1}}{\partial x}\left[\left(A\left(\xi_{t}\right)+\right.\right.\left(B\left(\eta_{t}\right)\right. \\
&+ {\left.\left.\left.\left[N_{1}\left(\xi_{t}, \eta_{t}\right) X_{j}, \ldots, N_{m}\left(\xi_{t}, \eta_{t}\right) X_{j}\right]\right) \mathcal{K}\left(\psi_{t}\right)\right) x\right] \leq 0 } \\
& \Psi(x)=\left\{x \in \mathbb{R}^{n} ; \mathcal{Q} x \leq \kappa\right\} \subseteq \operatorname{Co}\left\{X_{j}, j=1, \ldots, q\right\} \\
& \beta_{1}\left\|x_{t}\right\|^{2} \leq \mathcal{V}_{2}\left(x_{t}, \xi, \eta, \psi, t\right) \leq \beta_{2}\left\|x_{t}\right\|^{2} \\
& \\
& \mathcal{L} \mathcal{V}_{2}\left(x_{t}, \xi_{t}, \eta_{t}, \psi_{t}, t\right) \leq-\beta_{3}\left\|x_{t}\right\|^{2} \\
& \mathcal{D}_{0}=\left\{x \in \mathbb{R}^{n} ; \mathcal{V}_{1}\left(x_{t}\right) \leq \gamma^{-1}\right\} \subseteq \Psi(x)
\end{aligned}
$$

$\forall \xi_{t} \in Z, \eta_{t} \in S, \psi_{t} \in R$, where $\beta_{1}, \beta_{2}, \beta_{3}$ are real positive constants, then the gains $\mathcal{K}\left(\psi_{t}\right)$ and the Lyapunov level set $\mathcal{D}_{0}$ are solutions of problem 1.

Proof: The existence of matrix $\mathcal{Q}$, vectors $\kappa$ and $X_{j}$ satisfying relation (12) means that the vertices $X_{j}, j=1, \ldots, q$ allows to describe the convex hull of the polyhedral set $\Psi(x)$. Condition (15) means that, for each $\xi_{t} \in Z, \eta_{t} \in S, \psi_{t} \in R$, the set $\mathcal{D}_{0}$, defined from the definite positive function $\mathcal{V}_{1}\left(x_{t}\right)$ and the positive scalar $\gamma$, is included in the polyhedral set $\Psi(x)$. Then, for all $x_{t} \in \mathcal{D}_{0}, \forall t \geq 0$, there exist $\lambda_{j}\left(x_{t}\right) \geq 0, j=1, \ldots, q$, with $\sum_{j=1}^{q} \lambda_{j}\left(x_{t}\right)=1$, such that $\dot{x}_{t}$ can be computed by the polytopic model (10).

The satisfaction of the condition (11) means, by invoking convexity arguments, that:

$$
\begin{gathered}
\sum_{j=1}^{q} \lambda_{j}\left(x_{t}\right) \frac{\partial \mathcal{V}_{1}}{\partial x}\left[\left(A\left(\xi_{t}\right)+\left(B\left(\eta_{t}\right)+\left[N_{1}\left(\xi_{t}, \eta_{t}\right) X_{j}, \ldots, N_{m}\left(\xi_{t}, \eta_{t}\right) X_{j}\right]\right)\right.\right. \\
\left.\left.\mathcal{K}\left(\psi_{t}\right)\right) x\right]=\dot{\mathcal{V}}_{1}\left(x_{t}\right) \leq 0
\end{gathered}
$$

$\forall \xi_{t} \in Z, \eta_{t} \in S, \psi_{t} \in R$.

Hence, for each $\xi_{t} \in Z, \eta_{t} \in S, \psi_{t} \in R$, the time-derivative of $\mathcal{V}_{1}\left(x_{t}\right)$ along the trajectories of system (10) is negative or equal to zero along the trajectories of system (10). Therefore, the condition (11) guarantees the positive invariance of domain $\mathcal{D}_{0}$ w.r.t system (10). Conditions (13) and (14) ensure the stochastic exponential stability in the mean square sense of the system (10) (Srichander and Walker 1993).

Since model (10) allows to represent system (2) only in $\Psi(x)$, and by definition of $\mathcal{V}_{2}\left(x_{t}, \xi_{t}, \eta_{t}, \psi_{t}, t\right)$, $\mathcal{V}_{1}\left(x_{t}\right)$, and then the domain $\mathcal{D}_{0}$, we can conclude that the system (2) is locally exponentially stable in the mean square sense $\forall x_{0} \in \mathcal{D}_{0}$. Hence, the proof is complete.

Theorem 1 proposes a sufficient condition to solve the control design problem but such conditions appear not really constructive in order to exhibit suitable functions $\mathcal{V}_{1}\left(x_{t}\right), \mathcal{V}_{2}\left(x_{t}, \xi_{t}, \eta_{t}, \psi_{t}, t\right)$, matrices $\mathcal{K}\left(\psi_{t}\right)$ and $\mathcal{Q}$, vectors $X_{j}$ and $\kappa$. In order to develop available conditions numerically tractable, a first step consists in choosing:

$$
\begin{aligned}
& \text { 1) quadratic functions: } \\
& \qquad \mathcal{V}_{2}\left(x_{t}, \xi_{t}, \eta_{t}, \psi_{t}, t\right)=x_{t}^{T} \mathcal{P}\left(\xi_{t}, \eta_{t}, \psi_{t}\right) x_{t} \\
& \qquad \mathcal{V}_{1}\left(x_{t}\right)=x_{t}^{T} \mathcal{S} x_{t} \\
& \text { where } P\left(\xi_{t}, \eta_{t}, \psi_{t}\right)=P\left(\xi_{t}, \eta_{t}, \psi_{t}\right)^{T}>0 \text { and } \mathcal{S}=\mathcal{S}^{T}>0 . \\
& \text { 2) a matrix } \mathcal{Q} \text { defining } \Psi(x) \text { as } \mathcal{Q}=\left[\begin{array}{c}
I_{n} \\
-I_{n}
\end{array}\right] \in \mathbb{R}^{2 n \times n} .
\end{aligned}
$$

Lemma 2: (Mahout et al. 2002) The set $\Psi(x)$ defined by $\mathcal{Q}=\left[\begin{array}{c}I_{n} \\ -I_{n}\end{array}\right] \in \mathbb{R}^{2 n \times n}$ is such that:

- it admits $2^{n}$ vertices;

- these vertices are built from the ordered combinations formed from the components of the vector $\kappa$. We define this by:

$$
X_{j}=\operatorname{comb}\left(\kappa_{(i)}, i=1, \ldots, 2 n\right)
$$

Using such quadratic Lyapunov candidate functions $\mathcal{V}_{1}\left(x_{t}\right), \mathcal{V}_{2}\left(x_{t}, \xi_{t}, \eta_{t}, \psi_{t}, t\right)$ and such a matrix $\mathcal{Q}$, the following proposition derived from theorem 1 can be first considered.

Proposition 1: If there exists matrices $\mathcal{W}_{i j k}=$ $\mathcal{W}_{i j k}^{T}>0, \mathcal{S}=\mathcal{S}^{T}>0, \mathcal{K}_{k}$, vectors $X_{l}, l=$ $1, \ldots, 2^{n}$, and $\kappa$, and a positive scalar $\gamma$ satisfying

$$
\begin{aligned}
& A_{i}^{T} \mathcal{S}+\mathcal{S} A_{i}+\mathcal{K}_{k}^{T} \mathcal{N}_{i j l}^{T} \mathcal{S}+\mathcal{S} \mathcal{N}_{i j l} \mathcal{K}_{k} \leq 0 \\
& l=1, \ldots, 2^{n} \\
& {\left[\begin{array}{cc}
\mathcal{W}_{i j k} \tilde{A}_{i j k}^{T}+\tilde{A}_{i j k} \mathcal{W}_{i j k}+\mathcal{W}_{i j k} \mathcal{K}_{k}^{T} \mathcal{N}_{i j l}^{T}+\mathcal{N}_{i j l} \mathcal{K}_{k} \mathcal{W}_{i j k} & \mathcal{R}_{i j k}\left(\mathcal{W}_{i j k}\right) \\
\star & \mathcal{S}_{i j k}\left(\mathcal{W}_{i j k}\right)
\end{array}\right]<0} \\
& l=1, \ldots, 2^{n} \\
& \text { (20) } \\
& {\left[\begin{array}{cc}
\kappa_{d} \mathcal{S} & \mathcal{Q}_{d}^{T} \\
\mathcal{Q}_{d} & \gamma \kappa_{d}
\end{array}\right] \geq 0} \\
& d=1, \ldots, 2 n \\
& X_{l}=\operatorname{comb}\left(\kappa_{d}, d=1, \ldots, 2 n\right) \quad l=1, \ldots, 2^{n}
\end{aligned}
$$

$\forall i \in Z, j \in S$ and $k \in R$, where

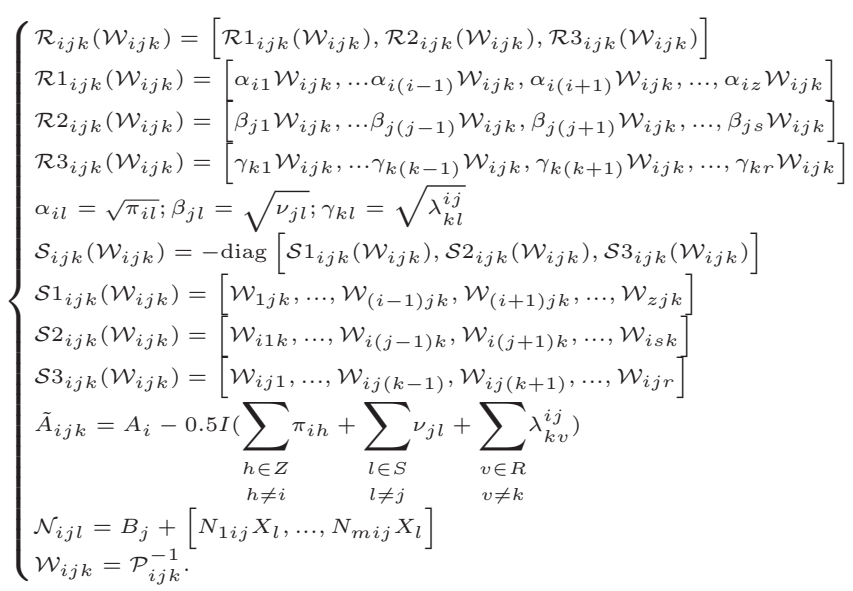

then the gain matrices $\mathcal{K}_{k}$ and the ellipsoidal Lyapunov level set $\mathcal{D}_{0}$ solve the problem 1 , where

$$
\mathcal{D}_{0}=\left\{x \in \mathbb{R}^{n} ; x^{T} \mathcal{S} x \leq \gamma^{-1}\right\}
$$

$\forall i \in Z, j \in S$ and $k \in R$.

Proof: The proof follows from that of theorem 1 by 
considering $\mathcal{V}_{1}\left(x_{t}\right)=x_{t}^{T} \mathcal{S} x_{t}$ and $\mathcal{V}_{2}\left(x_{t}, i, j, k, t\right)=$ $x^{T} \mathcal{W}_{i j k}^{-1} x$ with $\mathcal{W}_{i j k}=\mathcal{W}_{i j k}^{T}>0$ and $\mathcal{S}=\mathcal{S}^{T}>0$. The second step in the computation of numerically tractable solutions, uses proposition 1 and the reciprocal projection lemma to give more suitable sufficient conditions for the local stochastic stability of system (10). Such conditions are given by the following proposition:

Proposition 2: If there exist matrices $\mathcal{X}_{i j k}=$ $\mathcal{X}_{i j k}^{T}>0, \mathcal{X}=\mathcal{X}^{T}>0, \mathcal{K}_{k}, \bar{\Omega}_{i j k}, \bar{\Sigma}_{i j k}$, vectors $X_{l}$, $l=1, \ldots, 2^{n}$ and $\kappa$, and a positive scalar $\gamma$ satisfying

$$
\begin{aligned}
& {\left[\begin{array}{cccc}
\mu_{i j k} \mathbb{I}-\bar{\Omega}_{i j k}-\bar{\Omega}_{i j k}^{T} & \left(\mu_{i j k} \mathcal{X}_{i j k}-\bar{\Omega}_{i j k}\right) & \Theta_{i j k} & \mathcal{R}_{i j k}\left(\mathcal{X}_{i j k}\right) \\
\star & -\mu_{i j k} \mathbb{I} & 0 & 0 \\
\star & \star & -\mu_{i j k} \mathbb{I} & 0 \\
\star & \star & \star & \mathcal{S}_{i j k}\left(\mathcal{X}_{i j k}\right)
\end{array}\right]<0} \\
& l=1, \ldots, 2^{n} \\
& {\left[\begin{array}{crr}
\rho_{i j k} \mathbb{I}-\bar{\Sigma}_{i j k}-\bar{\Sigma}_{i j k}^{T} & \left(\rho_{i j k} \mathcal{X}-\bar{\Sigma}_{i j k}\right) & \Xi_{i j k} \\
\star & -\rho_{i j k} \mathbb{I} & 0 \\
\star & \star & -\rho_{i j k} I
\end{array}\right] \leq 0} \\
& \begin{array}{c}
{\left[\begin{array}{cc}
\kappa_{d} \mathcal{X} & \mathcal{X} \mathcal{Q}_{d}^{T} \\
\star & \gamma \kappa_{d}
\end{array}\right] \geq 0} \\
d=1, \ldots, 2 n
\end{array} \\
& X_{l}=\operatorname{comb}\left(\kappa_{d}, d=1, \ldots, 2 n\right), l=1, \ldots, 2^{n}
\end{aligned}
$$

$\forall i \in Z, j \in S$ and $k \in R$, where

$$
\left\{\begin{array}{l}
\Theta_{i j k}=\widetilde{A}_{i j k}+\mathcal{N}_{i j l} \mathcal{K}_{k}+\bar{\Omega}_{i j k} \\
\Xi_{i j k}=A_{i}+\mathcal{N}_{i j l} \mathcal{K}_{k}+\bar{\Sigma}_{i j k}
\end{array}\right.
$$

$\mu_{i j k}$ and $\rho_{i j k}$ are arbitrary positive scalars. These free scalar parameters are introduced to reduce the conservatism of the sufficient condition for exponential stochastic stability. Then the gain matrices $\mathcal{K}_{k}$ and the ellipsoidal Lyapunov level set $\mathcal{D}_{0}$ solve the problem 1, where

$$
\mathcal{D}_{0}=\left\{x \in \mathbb{R}^{n} ; x^{T} \mathcal{X}^{-1} x \leq \gamma^{-1}\right\}
$$

$\forall i \in Z, j \in S$ and $k \in R$.

Proof: The matrix inequality (18) (in proposition 1) can be written as follows:

$$
\tilde{A}_{i j k}^{T} \mathcal{P}_{i j k}+\mathcal{P}_{i j k} \tilde{A}_{i j k}+\mathcal{K}_{k}^{T} \mathcal{N}_{i j l}^{T} \mathcal{P}_{i j k}+\mathcal{P}_{i j k} \mathcal{N}_{i j l} \mathcal{K}_{k}+\Delta_{i j k}<0 \text { (29) }
$$

where

$$
\Delta_{i j k}=\sum_{\substack{h \in Z \\ h \neq i}} \pi_{i h} \mathcal{P}_{h j k}+\sum_{\substack{l \in S \\ l \neq j}} \nu_{j l} \mathcal{P}_{i l k}+\sum_{\substack{v \in R \\ v \neq k}} \lambda_{k v}^{i j} \mathcal{P}_{i j v}
$$

The use of the reciprocal projection lemma with $\Psi=\left(\sum_{\substack{h \in Z \\ h \neq i}} \pi_{i h} \mathcal{P}_{h j k}+\sum_{\substack{l \in S \\ l \neq j}} \nu_{j l} \mathcal{P}_{i l k}+\sum_{\substack{v \in R \\ v \neq k}} \lambda_{k v}^{i j} \mathcal{P}_{i j v}\right)$, and $S=\tilde{A}_{i j k}^{T} \mathcal{P}_{i j k}+\mathcal{K}_{k}^{T} \mathcal{N}_{i j l}^{T} \mathcal{P}_{i j k}$ yields $\left[\begin{array}{cc}\Delta_{i j k}+\mathcal{Z}_{i j k}-\left(\Omega_{i j k}+\Omega_{i j k}^{T}\right) & \mathcal{P}_{i j k} \tilde{A}_{i j k}+\mathcal{P}_{i j k} \mathcal{N}_{i j l} \mathcal{K}_{k}+\Omega_{i j k} \\ * & -\mathcal{Z}_{i j k}\end{array}<0\right.$ where $\mathcal{Z}_{i j k}$ is any given positive-definite matrix, $\forall i \in Z, j \in S$ and $k \in R$.

Let us define $\mathcal{X}_{i j k}=\mathcal{P}_{i j k}^{-1}$ and $\bar{\Omega}_{i j k}=\mathcal{X}_{i j k} \Omega_{i j k}$, then by the congruence transformation

$$
\left[\begin{array}{cc}
\mathcal{X}_{i j k} & 0 \\
0 & \mathbb{I}
\end{array}\right]
$$

and with a Schur complement operation with respect to the term

$$
\mathcal{X}_{i j k} \Delta_{i j k} \mathcal{X}_{i j k}
$$

the inequality (38) in turn becomes

$$
\left[\begin{array}{ccc}
\mathcal{X}_{i j k} \mathcal{Z}_{i j k} \mathcal{X}_{i j k}-\bar{\Omega}_{i j k} \mathcal{X}_{i j k}-\mathcal{X}_{i j k} \bar{\Omega}_{i j k}^{T} & \Theta_{i j k} & \mathcal{R}_{i j k}\left(\mathcal{X}_{i j k}\right) \\
\star & -\mathcal{Z}_{i j k} & 0 \\
\star & \star \mathcal{S}_{i j k}\left(\mathcal{X}_{i j k}\right)
\end{array}\right]_{(31)}<0
$$

Since, according to lemma $1, \mathcal{Z}_{i j k}$ can be any positive-definite matrix, we let $\mathcal{Z}_{i j k}=\mu_{i j k} I \forall i \in Z$, $j \in S$ and $k \in R$. Then, using the Schur complement operation with respect to the term

$$
\mathcal{X}_{i j k} \mathcal{Z}_{i j k} \mathcal{X}_{i j k}-\bar{\Omega}_{i j k}^{T} \mathcal{X}_{i j k}-\mathcal{X}_{i j k} \bar{\Omega}_{i j k}
$$

the inequality (39) can be further be written as

$$
\left[\begin{array}{cccc}
-\mu_{i j k}^{-1} \bar{\Omega}_{i j k} \bar{\Omega}_{i j k}^{T} & \left(\mu_{i j k} \mathcal{X}_{i j k}-\bar{\Omega}_{i j k}\right) & \Theta_{i j k} & \mathcal{R}_{i j k}\left(\mathcal{X}_{i j k}\right) \\
\star & -\mu_{i j k} \mathbb{I} & 0 & 0 \\
\star & \star & -\mu_{i j k} \mathbb{I} & 0 \\
\star & \star & \star & -\mathcal{S}_{i j k}\left(\mathcal{X}_{i j k}\right)
\end{array}\right]<0
$$

then, using the relation $-\mu_{i j k}^{-1} \bar{\Omega}_{i j k} \bar{\Omega}_{i j k}^{T} \leq-\bar{\Omega}_{i j k}-$ $\bar{\Omega}_{i j k}+\mu_{i j k} I$ yields the matrix inequality (32). Hence the feasibility of this last inequality, with respect to $\bar{\Omega}_{i j k}, \mathcal{X}_{i j k}, \mathcal{K}_{k}$ and $X_{l}$ is a sufficient condition to the feasibility problem of the matrix inequalitie (20), with respect to $\mathcal{W}_{i j k}, \mathcal{K}_{k}$ and $X_{l}$. Using same arguments as above, we can prove that the feasibility of matrix inequalities (33) and (34), with respect to $\bar{\Sigma}_{i j k}, \mathcal{K}_{k}, \mathcal{X}, X_{l}, \kappa$ and $\gamma$, implied the feasibility of matrix inequalities (19) and (21), respectively, with respect to the decision variables $\mathcal{S}, \mathcal{K}_{k}, X_{l}, \kappa$ and $\gamma$. Hence, the proof is complete.

Computational issues: It is important to note that the decision variables to be found by applying proposition 2 are $\bar{\Omega}_{i j k}, \bar{\Sigma}_{i j k}, \mathcal{X}_{i j k}=\mathcal{X}_{i j k}^{T}>0$, $\mathcal{X}=\mathcal{X}^{T}>0, \mathcal{K}_{k}, X_{l}, l=1, \ldots, 2^{n}, \kappa$, and $\gamma$. However, due to the presence of some terms involving products between some of these variables, inequalities (32)-(35) are nonlinear. This fact means that to solve constraints (32)-(35) as a feasibility problem is very hard or even impossible directly. A way to overcome this limitation consists in fixing, a priori, the value of some decision variables while searching the others. Moreover, since the implicit objective consists in enlarging the domain of stability $\mathcal{D}_{0}$, some convex optimization problem with LMI constraints can be considered. This is illustrated by the following algorithm: Algorithm 1:

- Step 0. Initialization: fix $\kappa_{d}, d=1, \ldots, 2 n$ and compute $X_{l}, l=$ $1, \ldots, 2^{n}$ from $(22)$.

- Step 1 .

- (Feasibility problem) Let $\gamma=1$. Compute $\mathcal{X}_{i j k}=$ $\mathcal{X}_{i j k}^{T}, \mathcal{X}=\mathcal{X}^{T}, \mathcal{K}_{k}, \bar{\Omega}_{i j k}$ and $\bar{\Sigma}_{i j k}, \forall i \in Z, j \in S$ and $k \in R$, solutions to the LMIs:

$$
\text { (32), (33), (34) }
$$

- Maximization of the volume of the domain of stability $\mathcal{D}_{0}$ ) Fix $\mathcal{X}$ and compute $\gamma$ solution to:

$$
\min \gamma
$$$$
\text { s.t }(34)
$$

- Step 2. (Maximization of the size of the compact domain $\Psi(x))$ Fix $\mathcal{X}_{i j k}, \mathcal{X}, \bar{\Omega}_{i j k}, \bar{\Sigma}_{i j k}$ and $\gamma$ and compute $\kappa_{d}, d=$ $1, \ldots, 2 n$ (and therefore $X_{l}, l=1, \ldots, 2^{n}$ ) solutions to:

$$
\min \left\{-\sum_{l=1}^{2^{n}} \varepsilon_{l}\right\}
$$




$$
\begin{aligned}
& \text { s.t } \quad(32), \quad(33), \quad(34), \quad(35) \\
& {\left[\begin{array}{cc}
\varepsilon_{l} & X_{l}^{T} \\
X_{l} & I_{n}
\end{array}\right] \geq 0, \quad l=1, \ldots, 2^{n}}
\end{aligned}
$$

- Step 3. Go to step 1 until no significant change in the volume of the initial condition set $\mathcal{D}_{0}$ has occurs.

\section{NUMERICAL EXAMPLE}

To illustrate the theoretical results presented above, let us consider a system with one possible fault in the plant components, i.e. $S=\{1,2\}$. The failure process is assumed to have Markovian transition characteristics. The FDI process is also Markovian with two states $R=\{1,2\}$. The following numerical values are used

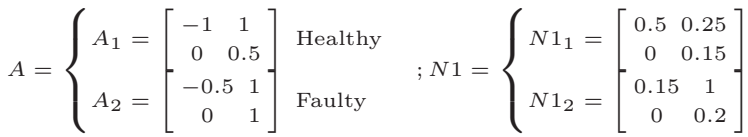

$$
\begin{aligned}
& N 2=\left\{\begin{array}{l}
N 2_{1}=\left[\begin{array}{cc}
0.2 & 0.5 \\
0 & 0.4
\end{array}\right] \text { Healthy } \\
N 2_{2}=\left[\begin{array}{cc}
0.15 & 0 \\
0 & 0.3
\end{array}\right] \text { Faulty }
\end{array} ; B=\left[\begin{array}{cc}
1 & 1 \\
-0.25 & 4
\end{array}\right]\right. \\
& {\left[\pi_{i j}\right]=\left[\begin{array}{cc}
-0.5 & 0.5 \\
0.1 & -0.1
\end{array}\right] ; \lambda_{i j}^{1}=\left[\begin{array}{cc}
-0.3 & 0.3 \\
1.2 & -1.2
\end{array}\right] ; \lambda_{i j}^{2}=\left[\begin{array}{cc}
-1.2 & 1.2 \\
0.4 & -0.4
\end{array}\right] \text {. }}
\end{aligned}
$$

Then, applying the algorithm 1, the LMI solutions yield the following parameters:

$$
\begin{gathered}
\mathcal{K}_{1}=\left[\begin{array}{cc}
-0.0192 & 0.1319 \\
-0.0969 & -0.6272
\end{array}\right] ; \mathcal{K}_{2}=\left[\begin{array}{cc}
-0.0188 & 0.1328 \\
-0.0983 & -0.6292
\end{array}\right] ; \\
\kappa=\left[\begin{array}{l}
9.1926 \\
3.9382 \\
3.9811 \\
3.3312
\end{array}\right] ; \gamma=0.1115 .
\end{gathered}
$$

Thus, we can guarantee that for any initial con-

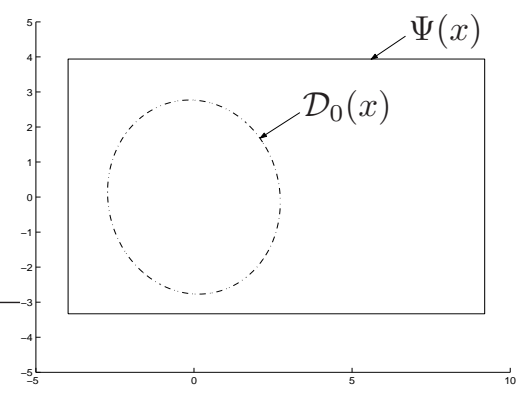

Figure 1. Domain of local exponential stochastic stability

ditions taken in $\mathcal{D}_{0}(x)$, defined from $\mathcal{X}$ and $\gamma$ (see Figure 1), the system (10) is locally exponentially stable in the mean square sense.

\section{CONCLUSION}

In this paper, we have considered the problematic of local exponential stochastic stabilization of continuous time BAFTCSMP. The design technique was based on a differential inclusion of the bilinear term for a restricted domain of the state space. The above problematic was addressed under a convex programming approach. Indeed, condition for local stochastic stability of the BAFTCSMP were derived and formulated through some matrix inequalities. The implicit problem being to maximize the region in which the closed-loop stability can be ensured, some convex optimization problems with LMI relaxations schemes were also stated. A numerical exemple was also included to illustrate and validate our theoretical results.

\section{REFERENCES}

Apkarian, P., H.D. Tuan and J. Bernussou (2001). Continuous-time analysis, eigenstructure assignment, and synthesis with enhanced linear matrix innequalities (lmi) characterizations. IEEE Transactions on Automatic Control 46, 1941-1946.

Costa, O. L. V., J. B. R. do Val and J. C. Geromel (1999). Continuous-time state-feedback $\mathrm{H}_{2^{-}}$ control of Markovian jump linear systems via convex analysis. Automatica 35(2), 259-268.

de Farias, D. P., J. C. Geromel, J. B. R. do Val and O. L. V. Costa (2000). Output feedback control of Markov jump linear systems in continuoustime. IEEE Transaction on automatic control 45(5), 944-949.

de Souza, C. E. and M. D. Fragoso (1993). $\mathcal{H}_{\infty}$ control for linear systems with markovian jumping parameters. Control Theory and Advanced Technology 9(2), 457-466.

Elliott, D. L. (1999). Bilinear systems. Encyclopedia of Electrical Engineering, Edited by John Webster, J. Wiley and Sons.

Ji, Y. and H. J. Chizeck (1990). Controllability, stabilizability, and continuous-time Markovian jump linear quadratic control. IEEE Transaction on automatic control 35, 777-788.

Ji, Y. and H. J. Chizeck (1992). Jump linear quadratic Gaussian control in continuous time. IEEE Transaction on automatic control 37(12), 1884-1892.

Mahmoud, M., J. Jiang and Y. Zhang (2003). Active Fault Tolerant Control Systems: Stochastic Analysis and Synthesis. Springer.

Mahout, V., S. Tarbouriech and G. Garcia (2002). Controller design for unstable uncertain bilinear systems. $10^{\text {th }}$ Mediteranean Conference on Control and Automation, Lisbon, Portugal.

Molchanov, A. P. and E. S. Pyatniskii (1989). Criteria of asymptotic satbility of differential and difference inclusions encountered in control theory. System and Control Letters 13, 59-64.

Srichander, R. and B. K. Walker (1993). Stochastic stability analysis for continuous-time fault tolerant control systems. International journal of control 57(2), 433-452. 